\title{
COMPLICAÇÕES ORBITÁRIAS DA MUCOCELE FRONTAL
}

\author{
ORBITAL COMPLICATIONS OF THE FRONTAL MUCOCELE
}

Natália Coelho Cavalcante, Mariana Florêncio Ferro, Taís Cassiano Bueno Vieira, Gleim Dias Souza, Luciana Rodrigues Quueiroz de Souza

DOI - 10.5935/2236-5117.2021v58a03

\section{RESUMO}

O presente estudo é uma revisão sistemática da literatura e tem como objetivo conceituar e compreender o mecanismo fisiopatológico da mucocele, com ênfase no comprometimento da órbita ocular pela mucocele do seio frontal, apontando os sinais e sintomas, complicações, quais os melhores exames de imagem e quais as opções de tratamento. Mucoceles são lesões da mucosa dos seios paranasais, de caráter benigno, expansivo e são causadas por uma obstrução dos óstios dos seios paranasais, que podem surgir devido a anormalidades congênitas, inflamação, infecção, alergia, traumas, cirurgias anteriores ou tumores. As mucoceles podem gerar comprometimento orbitário, devido ao seu caráter expansivo, somado a proximidade anatômica com a órbita, resultando em sintomas oftalmológicos como diplopia, redução da acuidade visual, perda de campo visual, proptose, ptose palpebral, edema periorbital, oftalmoplegia e deslocamento inferior do globo ocular.

Palavras-chave: Mucocele. Órbita. Seio Frontal

\section{ABSTRACT}

The present study is a systematic review of the literature and aims to conceptualize and understand the pathophysiological mechanism of mucocele, with emphasis on the ocular orbital involvement by the mucocele of the frontal sinus, pointing out its signs and symptoms, complications, the best imaging exams and the options of treatment. Mucoceles are lesions of the paranasal sinuses mucous membrane, with expansive and benign character, are caused by an obstruction of the paranasal sinuses ostia, which may arise due to congenital abnormalities, inflammation, infection, allergy, trauma, previous surgeries or tumors. Mucoceles can cause orbital involvement, due to their expansive nature, and anatomical proximity to the orbit, ophthalmological symptoms such as diplopia, visual acuity reduction, visual field loss, proptosis, palpebral ptosis, periorbital edema, ophthalmoplegia and inferior displacement of the eyeball.

Keywords: Mucocele. Frontal Sinus. Orbit
Natália Coelho Cavalcante - Universidade Católica de Brasília, Medicina Brasília - DF - Brasil.

Mariana Florêncio Ferro - Universidade Católica de Brasília, Medicina Brasília - DF - Brasil.

Taís Cassiano Bueno Vieira - Universidade Católica de Brasília, Medicina Brasília - DF - Brasil

Gleim Dias Souza - Universidade Católica de Brasília, Medicina - Brasília - DF Brasil. Instituto Hospital de Base, Radiologia - Brasília - DF - Brasil.

Luciana Rodrigues Queiroz de Souza - Instituto Hospital de Base, Radiologia - Brasília - DF - Brasil.

Correspondência: Natália Coelho Cavalcante. Quadra 10 Conjunto B, Sobradinho, Brasilia - DF, Brasil. CEP 73005102

Internet: nataliaccavalcante@gmail.com

Conflito de interesses: não existem conflitos de interesse.

\section{INTRODUÇÃO}

Mucoceles são lesões da mucosa dos seios paranasais de caráter benigno e expansivo, devido ao acúmulo de muco. Tem origem em situações que há obstrução dos óstios dos seios paranasais, como nas anormalidades congênitas, inflamação, infecção, alergia, traumas, cirurgias anteriores ou tumores. Em alguns casos, há evidência histológica de aumento no número de células secretoras na membrana de revestimento gerando uma hipersecreção de muco, o que pode ser um fator contribuinte. $1,2,3,4$

A obstrução dos seios paranasais, somada a produção e secreção contínua de muco, leva a distensão e erosão das paredes do seio. Por esse mecanismo, apesar de ter caráter benigno, tendem a se expandir, exercendo pressão, deslocando e destruindo estruturas próximas. A mucocele pode se expandir para a órbita, seios adjacentes, cavidade nasal, intracraniana ou através da pele. 0 caráter expansivo se torna possível em virtude do processo dinâmico de remodelação óssea. 5,6,7,8 
Esse tipo de lesão tem incidência semelhante em ambos sexos e pode ocorrer em qualquer idade, apesar de ser mais frequente na faixa etária dos 40 aos 70 anos. Os seios frontais são os mais acometidos pela mucocele, com $60 \%$ dos casos, seguidos pelos seios etmoidais e esfenoidal. 0 acometimento dos seios maxilares é extremamente raro.,10 Apesar de ser uma patologia cuja incidência é baixa, as potenciais complicações da mucocele são graves, e é ainda muito desconhecida entre médicos e profissionais da saúde, o que dificulta seu diagnóstico e resolução. Nesse sentido, o objetivo desse estudo é orientar quanto aos sinais e sintomas que poderiam aventar a hipótese diagnóstica de mucocele com complicações orbitárias, e a realizar diagnósticos diferenciais, principalmente com tumores.

\section{METODOLOGIA}

O presente estudo é uma revisão sistemática da literatura baseada em artigos disponíveis nas bases de dados Cochrane, PubMed (MEDLINE), Scielo, Bireme, LILACS e Google Acadêmico. Foram utilizados os descritores "mucocele", "frontal sinus mucocele", "mucoceles with intraorbital extension". Foram incluídos artigos completos e originais, artigos de revisão de literatura e relatos de casos, publicados em língua inglesa, portuguesa e espanhola. Foram excluídos capítulos de livros, cartas ao editor e trabalhos não publicados.

\section{DISCUSSÃO}

As mucoceles, geralmente, se expandem na direção onde há menor resistência. As lesões do tipo frontais podem apresentar uma variedade de sinais e sintomas devido ao efeito de massa e invasão de estruturas vizinhas, de modo que frequentemente há a destruição do osso mais fino da órbita ocular superior. A apresentação clínica varia de assintomática a cefaléia incapacitante e distúrbio visual. A proptose é o sinal mais comum de uma mucocele frontal, podendo ainda apresentar dor, cefaléia, assimetria facial, pressão maxilofacial, edema, epífora e exoftalmia resultantes do crescimento da lesão cística. Outros sintomas como ptose, restrição dos movimentos extra-oculares, diplopia, diminuição da acuidade visual, amaurose e perda do campo visual são decorrentes do seu caráter compressivo e, apesar de raro, consequente acometimento do II, III, IV e VI pares cranianos por contiguidade. .,6,8,11,12 $^{-12}$

Ocasionalmente, uma mucocele agressiva poderá causar destruição óssea, simulando uma neoplasia maligna. A erosão da parede anterior resulta em uma massa flutuante sob o periósteo do osso frontal, e nestes casos, a tomografia computadorizada (TC) é bastante útil para distinguir as duas patologias. Já a erosão da parede posterior pode acarretar em complicações como abscesso epidural, meningite, meningoencefalite, empiema subdural, abscesso cerebral, fístulas no líquido cefalorraquidiano (LCR) e convulsões. ${ }^{5,8,13,14}$

O diagnóstico de uma mucocele é baseado na história, exame físico e achados radiológicos. 0 aspecto radiográfico clássico de uma mucocele é o velamento do seio comprometido e afinamento generalizado das paredes do seio, bem como erosões ósseas. A mucocele geralmente é homogênea e sem ar. A tomografia computadorizada (TC) nos planos axial e coronal é utilizada como método de imagem de escolha para o diagnóstico, uma vez que consegue delinear a extensão da lesão e suas relações com as estruturas adjacentes. A TC usualmente demonstra massa expansiva, sem ar e o conteúdo da mucocele é evidenciado como uma substância homogênea de atenuação mucoide (10 a $18 \mathrm{HU})$. Normalmente, o contorno ósseo torna-se mais arredondado à medida que o osso é remodelado em resposta à crescente pressão dentro da cavidade sinusal. Além disso, pode ser observado afinamento gradual da parede do seio e erosões ósseas com esclerose. Existem três critérios para o diagnóstico por TC de uma mucocele: massa isodensa homogênea, margem claramente definida e osteólise irregular ao redor da massa. Raramente a administração de meio de contraste é necessária. $4,5,6,7,11$

A ressonância magnética (RM) pode auxiliar com informações adicionais da lesão e no diagnóstico diferencial de neoplasias e infecções. A RM exibe intensidades de sinal variáveis em imagens ponderadas em T1 e T2, dependendo do estado de hidratação, conteúdo proteico e viscosidade do conteúdo da mucocele. Caracteristicamente revelam um fino realce linear periférico com intensidade central de baixo sinal nas imagens ponderadas em $T 1 .{ }^{5,7,8}$

Para que ocorra sucesso no tratamento da mucocele, o processo que causa anormalidade na drenagem do seio frontal deve ser compreendido. 0 tratamento definitivo da mucocele frontal é cirúrgico, podendo apresentar diferentes abordagens, como a endoscópica e a cirurgia aberta. ${ }^{2,15}$

0 tratamento para correção da lesão era, no passado, feito através da técnica de Lynch, cuja abordagem aberta era muito utilizada, sendo uma incisão transcutânea na área superior medial da órbita. Essa técnica apresenta 
como vantagem a exposição de todo o seio, facilitando o reestabelecimento da drenagem, e além disso, tem menor incidência de lesões da duramater durante o processo de curetagem do seio. Entretanto, apresentava como desvantagens as cicatrizes na pele, as desinserções dos nervos e os danos ao saco lacrimal. A abordagem transcaruncular também é uma possibilidade, devido ao acesso rápido à órbita e a menor chance de lesão musculares e melhor resultado estético., ${ }^{5,16}$

Recentemente, a abordagem mais utilizada e considerada mais segura é a nasoendoscópica. Para essa técnica as complicações são raras, associado a bom resultado estético e baixa taxa de recidiva, pois é capaz de garantir a drenagem do seio. ${ }^{17,18}$

\section{RESULTADOS}

0 acometimento dos olhos pela mucocele frontal tem como sua complicação mais importante a perda da visão. Sua expansão gradativa e caráter destrutivo das paredes ósseas conferem uma gama de sinais e sintomas decorrentes principalmente da compressão do globo ocular. 0 sinal mais evidente, relacionado proporcionalmente à extensão da mucocele, é a proptose que pode vir acompanhada de outros sintomas. Os pacientes acometidos podem evoluir de várias formas, com associações diferentes de apresentações como: tumoração em região orbitária superior, destruição óssea adjacente, deslocamento em direção inferior e lateral da órbita, restrição na mobilidade ocular, oftalmoplegia, diminuição da acuidade visual, dor, cefaleia, epífora, atrofia de nervo óptico, diplopia e amaurose. ${ }^{19,20,21}$

A formação de um abscesso, chamada mucopielocele, também é uma complicação importante que pode acarretar em outras repercussões além dos sinais e sintomas compressivos comuns. São vários os fatores que predispõem o paciente a desenvolver uma infecção da mucocele, dentre os quais podemos citar: processos alérgicos recorrentes, sinusite crônica, cirurgia ou traumatismo facial prévio, ou qualquer alteração que promova obstrução dos óstio sinusais, como uma neoplasia por exemplo. Porém em até um terço dos casos de mucopielocele não é possível identificar o fator predisponente desses pacientes. 0 processo infeccioso e inflamatório próximo à região orbitária poderá causar então por contiguidade várias alterações como: edema periorbitário, hiperemia, dor, flutuação, edema de papila, neurite óptica, celulite e dor intensa., 52,22

A tomografia computadorizada é o exame de escolha. Destaca-se na identificação da mucocele e do tamanho de sua extensão, e ao revelar com melhor exatidão o acometimento ósseo devido ao seu caráter erosivo. Já a ressonância magnética se sobressai no nível de detalhamento da invasão de outros tecidos próximos, pois é caracterizada por diferenciar melhor as densidades variáveis das partes moles, e quando associada ao uso de contraste possui grande valor na exclusão de neoplasias. Portanto, para avaliar com mais qualidade a extensão do acometimento orbitário, existe um ganho no uso da ressonância magnética. ${ }^{20,23,24,25}$

0 tratamento mais indicado é o cirúrgico pela via nasoendoscópica com marsupialização da mucocele, com objetivo de reestabelecer a drenagem adequada dos seios frontais. Demonstrou-se que a técnica endoscópica, além de proporcionar um resultado estético mais satisfatório para o paciente, também apresenta menor morbidade e taxa de recidiva bastante diminuída. 8,18,26,27

\section{CONCLUSÃO}

A mucocele é uma doença de comportamento biológico benigno, cuja localização mais comum é a do tipo frontal, e que esta, devido à sua topografia, frequentemente se expande e acomete estruturas adjacentes como a região da órbita ocular, resultando então em uma série de sinais e sintomas característicos dessa complicação. É importante ressaltar que a investigação das mucoceles está indicada sempre que houverem sintomas oculares como proptose, diplopia, oftalmoplegia, amaurose e redução da acuidade visual, associados a história de trauma, sinusite crônica ou cirurgias prévias que envolveram os seios da face.

A tomografia computadorizada, dentre os métodos de detecção, é um exame de excelência no diagnóstico inicial e no manejo posterior da mucocele, revelando principalmente a invasão de paredes ósseas. Já a ressonância magnética se mostrou superior no estudo complementar da lesão, pois evidencia melhor a sua extensão e invasão de outras estruturas moles como a órbita, visto que é possível detectar as diferentes densidades dos tecidos neste exame de imagem. A cirurgia por meio da via nasoendoscópica com marsupialização da mucocele revelou-se como a mais efetiva na correção do quadro, demonstrando menos complicações e recidivas e melhor resultado estético.

Em suma, o presente estudo demostra a importância de um diagnóstico precoce da mucocele visando minimizar os prejuízos do paciente, tendo em vista que as 
complicações podem gerar consequências graves como a perda da visão.

\section{CONFLITOS DE INTERESSES}

Não houve conflitos de interesses durante a confecção deste artigo.

\section{REFERÊNCIAS}

1. Fernandes $R$, Pirgousis $P$ : Longstanding giant frontal sinus mucocele resulting in extra-axial proptosis orbital displacement and loss of vision. J Oral Maxillofac Surg 68:3051, 2010

2. Park C, Stoffella E, Gile J, et al: Osteoplasty flap technique for repair of latent (30-year) post-traumatic frontal sinus mucocele: Case report and review of the literature. J Oral Maxillofac Surg 70:2092, 2012

3. Pierse J, Stern A: Benign cysts and tumors of the paranasal sinuses. Oral Maxillofac Surg Clin North Am 24:249, 2012

4. Lloyd G, Lund V], Savy L, Howard

5. D. Radiology in Focus: Optimum imaging for mucoceles. The Journal of Laryngology and Otology. 2000; 114: 233 236

6. Cagigal BP, Lezcano JB, Blanco RF, Cantera JM, Cuéllar LA, Hernández VA. Frontal sinus mucocoele with intracranial and intraorbital extension. Med Oral Patol Oral Cir Bucal. 2006; 11: E527-30.

7. Aggarwal SK, Bhavana K, Keshri A, Kumar R, Srivastava A. Frontal sinus mucocele with orbital complications: Management by varied surgical approaches. Asian Journal of Neurosurgery. 2012;7(3):135-140.

8. Belli S, Oktay MF. Bilateral frontal sinus mucocele: Histopathological and clinical review of a case. Medical Science and Discovery, 2016; 3(1): 55-9

9. Santos PLD, Chihara LL, Alcalde LFA, Masalskas BF, Sant'Ana E, Faria PEP. Outcomes in Surgical Treatment of Mucocele in Frontal Sinus. Journal of Craniofacial Surgery. 2017; 28(7): 1702-1708.

10. Carmichael RA, Kang DR. Frontal Sinus Mucopyocele Presenting as a Subcutaneous Forehead Mass. J Oral Maxillofac Surg. 2015;73:2155-61.

11. Obeso S, Llorente JL, Rodrigo JP, et al: Paranasal sinuses mucoceles: Our experience in 72 patients. Acta Otorrinolaringol Esp 60:332, 2009

12. Mehta AA, Wagner LH, Blace N. Spontaneous upper eyelid ecchmosis: A rare presenting sign for frontal sinus mucocele.

13. Melo $A A$, Neto $C$, da Silva $S$, Gouveia $M C L$, Santos PF. Amaurosis secondary to sphenoid mucocele. Arquivos Internacionais de Otorrinolaringologia. 2011; 15(4): 523 525.

14. Mourouzis C, Evans B, Shenouda E: Late presentation of a mucocele of the frontal sinus: 50 Years postinjury. J Oral Maxillofac Surg 66:1510, 2008

15. Mohan S. Frontal Sinus Mucocele with Intracranial and Intraorbital Extension: A Case Report. J. Maxillofac. Oral Surg. 2012; 11(3): 337-339.
16. Bell R, Dierks E, Brar P, et al: A protocol for the management of frontal sinus fractures emphasizing sinus preservation. ] Oral Maxillofac Surg 65:825, 2007

17. Wang TJ, Liao SL, Jou JR, et al: Clinical Manifestations and Management of Orbital Mucoceles: the Role of Ophthalmologists. Jpn J Ophthalmol 2005;49:239-245

18. Stokken J, Wali E, Woodard T, et al: Considerations in the management of giant frontal mucoceles with significant intracranial extension: A systematic review. Am J Rhinol Allergy 30, 301-305, 2016.

19. Seredyka-Burduk M, Burduk PK, Wierzchowska M, Kaluzny B, Malukiewicz. Complicações oftálmicas da cirurgia endoscópica dos seios nasais. Braz. j. otorhinolaryngol. 2017; 83(3): 318-323.

20. Gupta S, Goyal R, Shahi M. Frontal sinus mucopyelocele with intracranial and intraorbital extension. Nepalese Journal Of Ophthalmology. 2011; 3(1): 91-92.

21. Severino R, Severino P. Fronto- orbital mucocele with intracranial extension: a case report. J Surg Case Rep. 2017; 6: 1-3.

22. Soares M, Mota S, Gabriel T, Guimarães A, Freire F, Henriques $P$ et al. Mucopiocelo fronto-etmoidal, a propósito de um caso clínico. Revista Portuguesa de Otorrinolaringologia e Cirurgia Cérvico-Facial 2014; 52: 235-238.

23. Aydin E, Akkuzu G, Akkuzu B, Bilezikci B. Frontal mucocele with an accompanying orbital abscess mimicking a frontoorbital mucocele: case report. BMC Ear Nose Throat Disord. 2006; 6:6.

24. Rayes TR, Beraldin BS, Rayes A, Rayes GR. Mucocele Etmoidal e Frontoetmoidal com extensao orbitária: Relato de dois casos. Arquivos Catarinenses de Medicina 2008; 37: 87-90.

25. Martos-Fernández M, Sahuquillo- Barris J, MaletHernández D. ¿Cuál es su diagnóstico?. Rev Esp Cirug Oral y Maxilofac. 2017; 39(1): 53-54.

26. Carvalho BV, Lopes ICC, Corrêa JB, Ramos LFM, Motta EGPC, Diniz RLFC. Apresentações típicas e atípicas de mucocele dos seios paranasais na tomografia computadorizada. Radiol Bras. 2013; 46(6): 372-375.

27. Beigi B, Vayalambrone D, Kashkouli MB, Prinsley P, Saada J. Combined external and endonasal approach to frontoethmoidal mucocele involving the orbit. Journal of current ophthalmology. 2016; 28(1): 37-42.

28. Erdogan BA, Unlu N, Aydin S, Avci H. Frontal Mucocele Extended Orbita and Endoscopic Marsupialization Technique. Journal of Craniofacial Surgery. fev. 2018. 


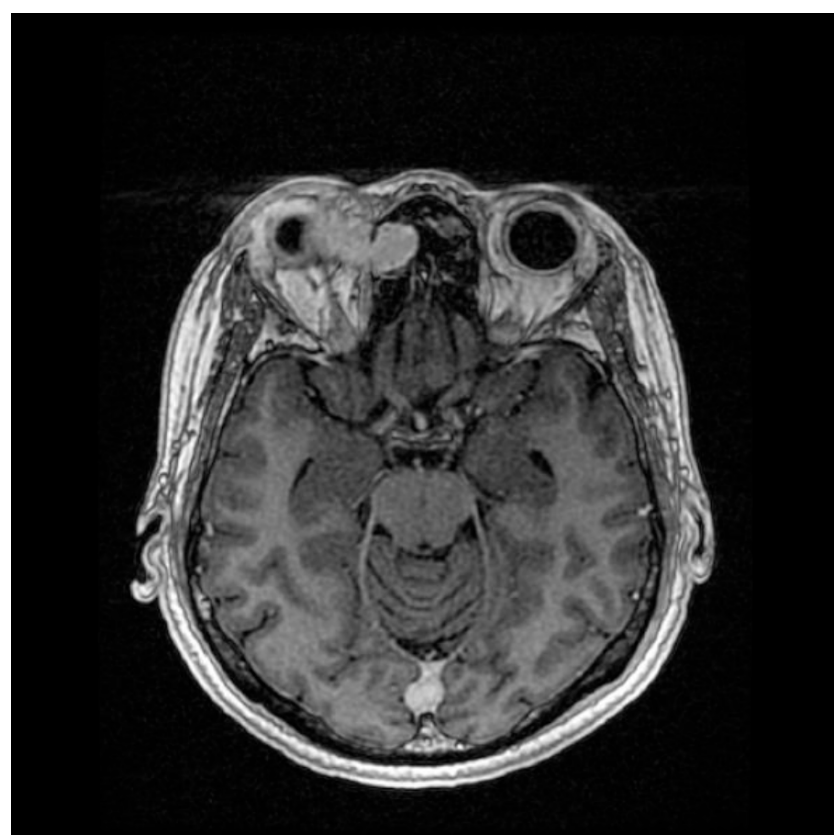

Figura 1. Ressonância Magnética, sequência axial T1 pós-contraste demonstrando deiscência da parede lateral da órbita à altura das células etmoidais mediais com protrusão do conteúdo para o sei interior exercendo compressão do músculo reto-lateral e da gordura retro bulbar

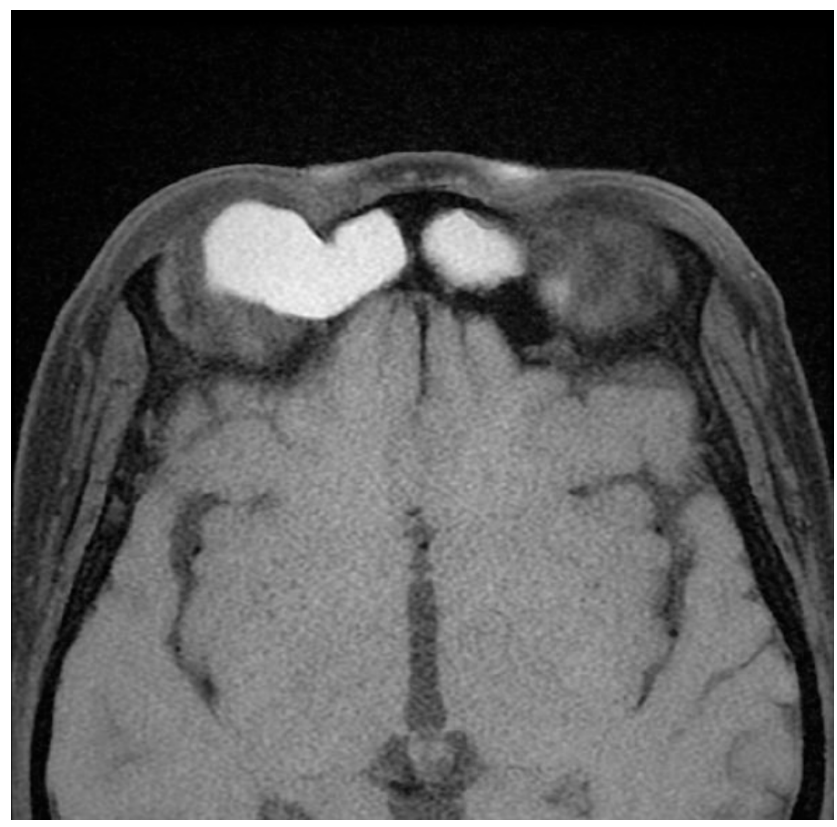

Figura 2. Ressonância Magnética, Sequência axial T1 FSE pré-contraste observando-se material de intensidade de sinal aumentada ocupando o seio frontal, com deiscência de sua parede e migração do conteúdo para o interior da órbita.

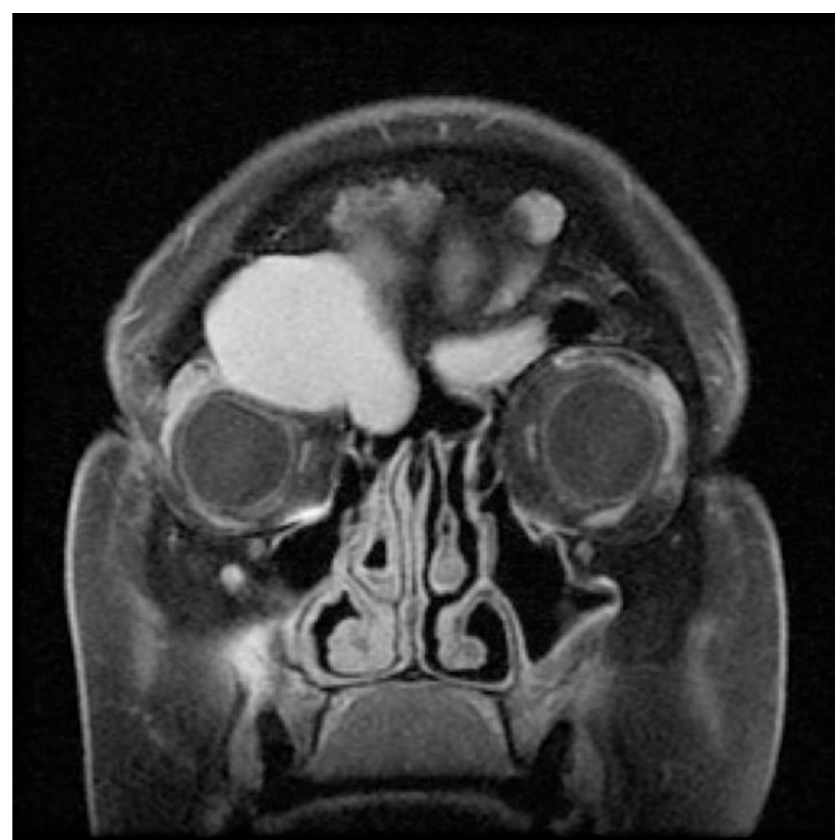

Figura 3. Ressonância Magnética, Sequência coronal T1 FSE com contrate, constata-se ruptura da fóvea etmoidal com invaginação de seu conteúdo, comprimindo o músculo reto-superior e o globo ocular. Há também ruptura da parede superior e posterior do seio frontal a direita com compressão do parênquima encefálico. 